\title{
High-Definition Video Distribution in 802.11g Home Wireless Networks
}

\author{
Víctor Miguel, Julián Cabrera, Fernando Jaureguizar, Narciso García \\ Universidad Politécnica de Madrid, Spain
}

\begin{abstract}
Multimedia distribution through wireless networks in the home environment presents a number of advantages which have fueled the interest of industry in recent years, such as simple connectivity and data delivery to a variety of devices. Together with High-Definition (HD) contents, multimedia wireless networks have been proposed for several applications, such as IPTV and Digital TV distribution for multiple devices in the home environment. For these scenarios, we propose a multicast distribution system for High-Definition video over 802.11 wireless networks based on rate-limited packet retransmission. We develop a limited rate ARQ system that retransmits packets according to the importance of their content (prioritization scheme) and according to their delay limitations (delay control). The performance of our proposed ARQ system is evaluated and compared with a similarly rate-limited ARQ algorithm. The results show a higher packet recovery rate and improvements in video quality for our proposed system.
\end{abstract}

\section{INTRODUCTION}

Wireless networks based on the 802.11 standard are becoming increasingly important for home video distribution systems [1][2]. Their application in the home environment brings multimedia home devices, such as Digital TV decoders and IPTV set-top boxes, into a wireless network. This infrastructure enables the reception of the video stream through a variety of wireless devices in different locations.

At the same time, more multimedia contents are broadcast in High-Definition (HD) format in order to enhance user experience. Their distribution through wireless networks has also been the object of several studies in recent years [3][4].

Some of these works are focused on distribution of HD Digital Television (DTV) [5] to several users in the home environment. Multicast enables simultaneous distribution to several devices but also presents several challenges. Multicast transmission of multimedia contents through wireless networks is specially challenging, since the changing behavior of the wireless channel causes random packet losses and no MAC level retransmissions are available.

In this paper we propose a multicast distribution system for High-Definition video contents through 802.11 wireless networks in home environments. This system incorporates retransmissions for packet loss compensation with a limited retransmission rate. Its performance is enhanced through a packet priority scheme and a delay control mechanism.

This work has been partially supported by Alcatel-Lucent and the Spanish Administration agency CDTI under project CENIT-VISION 2007-1007 and by the Ministerio de Ciencia e Innovacion of the Spanish Government under project TEC2007-67764 (SmartVision).

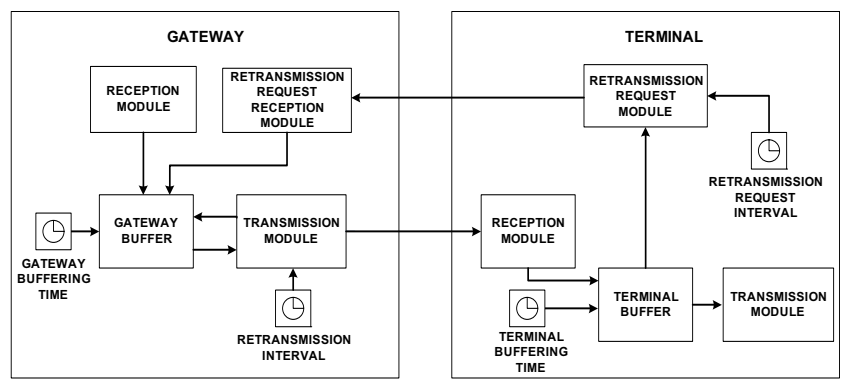

Fig. 1 Functional Diagram of the System

\section{SYSTEM DESCRIPTION}

The architecture of our proposed system consists of two entities, the Video Gateway and the Terminal, as shown in Figure 1. The Video Gateway streams the HD video to the Terminals through the wireless link and also includes the retransmission subsystem. This subsystem stores the RTP packets during a certain Gateway Buffering Time and receives Retransmission Request packets from the Terminals, retransmitting requested RTP packets with a frequency given by the Retransmission Interval. After this Buffering Time has expired for a packet, the Gateway erases it from the buffer.

Each Terminal receives the RTP packets, storing them for a certain Terminal Buffering Time, and uses their RTP sequence numbers to detect and locate lost packets. Then, each Terminal sends a Retransmission Request packet to the Gateway with a frequency set by the Retransmission Request Interval. Terminals receive the retransmissions and store them in the buffer. Once their Terminal Buffering Time has expired, the packets are sent from the Terminal buffer to the video player.

The Terminal Buffering Time determines the maximum waiting time for retransmission and the latency of the video transmission. Any retransmissions arriving outside this waiting interval will be ignored and designated as late arrivals.

Our proposed system adds intelligence to the retransmission process through the joint implementation of prioritization and delay control processes. These mechanisms improve the performance of rate-limited ARQ systems by arranging which packets must be retransmitted in order to increase the efficiency of the limited retransmission rate and enhance the quality of the received video.

\section{A. Prioritization Scheme}

The prioritization scheme is based on the importance of each packet according to their video content. The proposed system assigns priorities using Alcatel-Lucent's RTP rewrapper [7]. This tool packetizes the RTP flow so that every RTP packet only contains information from one video frame. 
It also provides information about the content of the packet through RTP extension headers.

The packets containing information from an Instantaneous Decoding Refresh frame (IDR frame) are considered the highest priority, since their loss has an impact on the entire group of pictures (GOP). The packets belonging to reference frames are the next in priority as these frames are used to encode other frames, which would also be affected by their loss. Finally, the packets belonging to non-reference frames are attributed the lowest priority.

Within each priority class, those packets belonging to the beginning of the frame are assigned the highest priority because, if these packets are lost, the associated frames are not decoded at the receiver.

\section{B. Delay Control}

One of the drawbacks of retransmissions in wireless systems is the possibility of late arrivals, which do not contribute to information recovery and waste retransmission rate. This is especially disadvantageous in the case of rate-limited systems.

In order to reduce the number of late arrivals and make a more efficient use of the retransmission rate, the system uses RTP timing information from the video flow in order to estimate the Round Trip Time (RTT) between each Terminal and the Gateway. This estimation is carried out at the Gateway through information contained in the Retransmission Requests from each Terminal.

Thanks to these RTT estimations, the Gateway is able to determine which retransmitted packets would be late arrivals for all the terminals and avoids their retransmission, saving their share of the limited rate for successful retransmissions.

\section{RESULTS}

The system is evaluated within the latency requirements specified in [6] for wireless distribution of HD Television, using a Terminal Buffer Time of 200 milliseconds. The transmitted video flow consists of H.264 video packetized in RTP/MPEG2-TS. The transmitted contents are 1080p, with an average throughput of $10 \mathrm{Mbps}$ and peaks of up to $15 \mathrm{Mbps}$.

The evaluation scenario is a multicast video distribution to two wireless Terminals, using a simulated channel model with a packet loss rate of $2 \%$ and a mean error burst length of 20 packets to connect the Video Gateway with the two Terminals. The values for these parameters are based on our transmission experiments carried out in the home environment.

We compare our proposed ARQ system with a rate-limited ARQ system setting the maximum retransmission rate as $5 \%$ of the original video flow. The evaluation of both systems is carried out in terms of the overall packet recovery rate, defined as the percentage of recovered packets in relation to the amount of lost packets, and the retransmission efficiency rate, defined as the percentage of successful retransmissions in relation to the total amount of retransmissions. We also measure the quality of the received video for both ARQ systems through its PSNR.
TABLE I

RESULTS FOR BUFFER TERMINAL TIME $=200 \mathrm{MS}$

\begin{tabular}{|c|c|c|}
\hline & $\begin{array}{c}\text { Rate-limited } \\
\text { ARQ }\end{array}$ & $\begin{array}{c}\text { Proposed } \\
\text { ARQ }\end{array}$ \\
\hline \multicolumn{3}{|l|}{ User 1} \\
\hline Overall Recovery Rate & $33,8 \%$ & $40,1 \%$ \\
\hline Retransmission Efficiency Rate & $26,6 \%$ & $35,7 \%$ \\
\hline PSNR & $28,6 \mathrm{~dB}$ & $34 \mathrm{~dB}$ \\
\hline \multicolumn{3}{|l|}{ User 2} \\
\hline Overall Recovery Rate & $14,7 \%$ & $17,8 \%$ \\
\hline Retransmission Efficiency Rate & $19,8 \%$ & $25 \%$ \\
\hline PSNR & $28,46 \mathrm{~dB}$ & $29,8 \mathrm{~dB}$ \\
\hline
\end{tabular}

The results in Table I show that our system achieves a higher recovery rate and retransmission efficiency rate thanks to the delay limitation mechanism in both users. User 2 shows an average delay higher than User 1, which explains the difference in the results. While the rate-limited ARQ system may waste a portion of the limited rate in failed retransmissions, our proposed system makes smarter retransmission decisions, achieving higher recovery and efficiency rates. The intelligence of our proposed system can also be noted in the results in the PSNR, which are improved thanks to the benefits of the prioritization process.

\section{CONCLUSIONS}

Wireless home distribution of HD video in services such as IPTV and DTV to multiple devices can be carried out through multicast transmission. In order to solve the challenges presented by these scenarios, we have proposed and implemented a rate-limited ARQ-based system with intelligent, delay-controlled retransmissions for multicast distribution of High-Definition video over 802.11 wireless networks.

Our preliminary results, obtained in a multicast scenario with several users, show that our proposed ARQ performs better than a similarly rate-limited ARQ scheme. Our proposed system is proved to make a better use of the available retransmission rate thanks to the delay control. In addition, the prioritization scheme also improves video PSNR.

\section{REFERENCES}

[1] R. Schmitt and R.Y. Chen, "Wireless Home Entertainment Networking using IEEE 802.11e QoS WLAN", in IEEE Transactions on Consumer Electronics, January 2005, pp. 305-306.

[2] S. Park, R. Haw, C. S. Hong, "System architecture for IPTV seamless service in mobility", in International Conference on Consumer Electronics, January 2010, pp. 59-60.

[3] G. Lawton, "Wireless Video HD Video Heats Up", IEEE Computer, vol. 41, no. 12, pp. 18-20, December 2008.

[4] P. Westergaard, "High-Definition Video QoS Over a Wireless 802.11 Network in an Integrated System", in IEEE Transactions on Consumer Electronics, February 2006, pp. 447-448.

[5] T. Shida, T. Sato, K. Tsunashima, H. Kosaka and K. Sugiyama, "Robust HD Video Stream Transmission for Wireless DTV", in IEEE Transactions on Consumer Electronics, February 2007, pp. 96-99.

[6] “Wireless LANs Usage Models", IEEE 802.11-03/802r23, IEEE 802.11 Group

[7] D. C. Robinson and A. Villegas, "Intelligent wrapping of video content to lighten downstream processing of video streams", European Patent EP2071850, 2009. 